\title{
A CASE OF SYSTEMIC TORULA INFECTION WITH TUMOUR FORMATION IN THE MENINGES
}

\author{
BY \\ L. KRAINER, J. M. SMALL, A. B. HEWLITT, and T. DENESS
}

(Received 28Th August, 1946)

THE case described below showed features distinct from the common clinical and pathological picture of torula infection. Systemic lesions were prominent before signs of central nervous system involvement. The lesion in the cranium gave rise to the clinical picture of a space-occupying lesion, while resection of the tumour was carried out with some measure of success.

Typical cases of torula meningo-encephalitis show diffuse, patchy meningitis with perivascular and embolic extension into the brain substance, while tumour formation in the meninges is rare (Swanson and Smith, 1944) and systemic lesions are usually inconspicuous. The causative organism is a spheroidal, encapsulated, yeast-like fungus, its classification being more often based on morphological than on biological features, as the diagnosis is usually established only after histological examination of necropsy material.

The term "Torula histolytica" (Stoddard and Cutler, 1916), commonly used in the literature, was originally applied to an organism isolated from the lung lesion of a horse; but according to Dodge (1935) the causative organism is a species of the genus cryptococcus (type species, Cryptococcus mollis: Kuetzing, 1833). Freeman (1930) distinguished three types of Torula histolytica. The first was an extremely small organism, 3 to 5 microns in diameter, seen in a fatal case; the second was a larger organism, about 15 microns in diameter; and the third was an organism measuring from 15 to $\mathbf{4 0}$ microns, found in cases with purely meningeal lesions. The cultural and biological behaviour of the organism was extensively studied by Nino (1929 and 1938).

\section{Case History}

The patient, a Sikh, aged 32, was admitted to hospital on Aug. 9, 1944, with a simple fracture of the forearm. While in hospital he complained of pain over the left supra-orbital margin for which no cause could be found. Three weeks later, however, a swelling had appeared just below the supra-orbital margin. This was incised and blood-stained gelatinous material evacuated, but a month later it had recurred and was again evacuated. On Nov. 9 he was said to have had an epileptiform attack. In the middle of January, 1945, another such attack occurred, characterized by clonic movements of the right leg.

. On admission to No. 7 I.B.G.H. (IT), Kirkee, in February, 1945, a discharging wound was present beneath the left supra-orbital margin, deep to which was a mass displacing the orbital contents (Fig. 1). Radiological examination revealed erosion of the inner and superior orbital walls with involvement of the frontal sinus. The Wassermann reaction was negative, urine examination showed nothing abnormal, and a biopsy on Feb. 27 was inconclusive. In March a fluctuant mass $5 \mathrm{~cm}$. by $3 \mathrm{~cm}$. resembling a cold abscess appeared over the right femoral trochanter and thigh, but radiographs of the hip joint, femur, and pelvis were normal. Aspiration produced

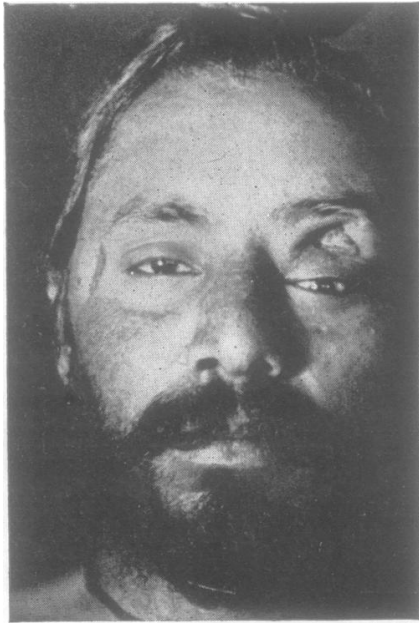

FIG. 1.-Showing the orbital lesion. a turbid, faintly yellow, viscous fluid, from a smear of which a diagnosis of cryptococcal infection was made by Major L. Krainer. On the second of March aspiration was repeated and a swab taken from the orbital swelling, the material being used for bacteriological examination and animal inoculation. The organism seen in the fluid from the thigh lesion was also found in material obtained from the orbital wound. The patient was given massive doses of iodide, and the thigh lesion subsided within five weeks, but there was no clinical improvement of the orbital lesion. Exploration was decided upon and was performed by Major A. B. Hewlitt on June 14, 1945. Masses of granulation tissue were removed from the orbit, left frontal, and ethmoid sinuses after removal of the anterior wall of the frontal sinus; the roof of the left frontal sinus was found to be eroded (Fig. 2) and the dura mater was visible in an area about an inch in diameter. The wound cavity was drained and was granulating satisfactorily by the end of July, being completely healed 


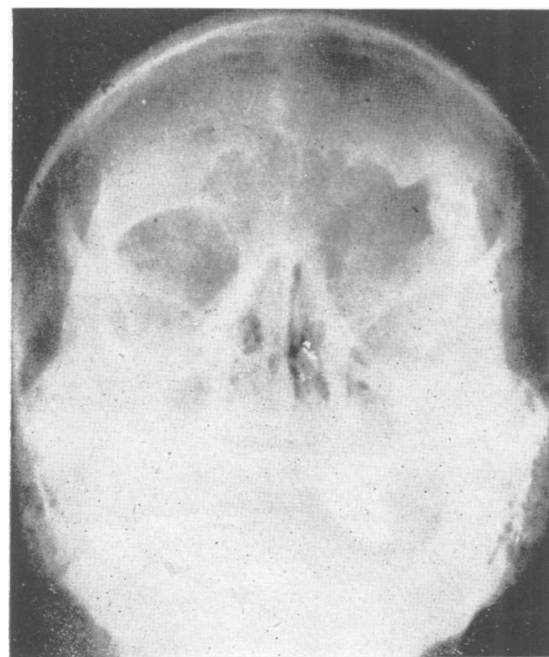

FIG. 2.-Showing the defect of the left supraorbital plate.

by the end of August. A radiograph of the chest revealed a suspicious shadow in the right upper lobe (? tuberculosis ? blastomycosis), but despite the administration of iodide the patient had no sputum, so that the nature of the lesion remained undisclosed. Repeated examination of the fæces failed to show the organism.

On Oct. 3, 1945, the patient had a generalized epileptiform convulsion, shortly after which the abdominal reflexes were noted to be absent on the right side, the right knee- and ankle-jerks were increased and there were bilateral extensor plantar responses. The patient stated that before the attack he had experienced a numb feeling in the right leg. By the end of October he was complaining of headache and was drowsy; examination show marked papillœdema, increased reflexes in the right leg, and absent abdominal reflexes on the right side. At this time the cerebrospinal fluid pressure was $400 \mathrm{~mm}$. but the cell count and biochemistry of the cerebrospinal fluid were normal, no torulæ being seen. A diagnosis of left frontal lobe involvement by tumour or blastomycetic infection was made.

Operation (Major I. M. Small) Oct. 10, 1945.

Preliminary ventriculography showed a marked shift of the ventricular system to the right with maximal shift and foreshortening of the frontal horn on the left side (Figs. 3 and 4). On the same day a left frontal osteoplastic flap was turned, the bone being found to be vascular and the dura tense on exposure. Subfrontal extra-dural exploration showed no abnormality apart from avascular adhesions in the vicinity of the defect of the supraorbital plate. The dura was opened over the frontal tip, disclosing an abnormal surface, the resistance being such that a brain needle could not be introduced. The dura mater was opened more extensively, disclosing a normal cortex abutting on the abnormal surface, and the former could be easily separated from the tumour which now appeared exactly like an anteriorly placed subfrontal meningioma. Using the diathermy loop the tumour was degutted, and when it was completely removed it was found to be about the size of a tennis ball, and had a dural attachment about $1 \frac{1}{4}$ in. in diameter in the subfrontal dura overlaying the old defect in the supra-orbital plate. This was completely removed, together with a surrounding fringe of normal dura. The line of demarcation between tumour and brain was distinct throughout; on one occasion only was a small quantity of mucoid material seen within the tumour which otherwise closely resembled a meningioma. The resulting cavity, exposing the falx medially, was filled with $200 \mathrm{c.cm}$. of a solution containing 100 units of sodium penicillin per c.cm., the dura mater was then closed and the flap replaced with an extradural drain.

The wound healed by primary intention, and although the cerebrospinal pressure remained at a level of $300 \mathrm{~mm}$. for about 10 days, at the end of three weeks it had fallen to $150 \mathrm{~mm}$., the cytology and biochemistry being normal. Culture of the cerebrospinal fluid on two occasions was sterile. The papillœdema rapidly subsided, and slightly increased knee- and ankle-jerks on the right side were the only remaining signs in the central nervous system.

The patient presented himself in good condition in September, 1946. The only neurological signs were exaggerated knee and ankle jerks on the right side.

\section{Pathology}

Direct examination (aspiration from the swelling in the right thigh).-A smear stained with Gram's method showed large Gram-positive spheroidal cells with

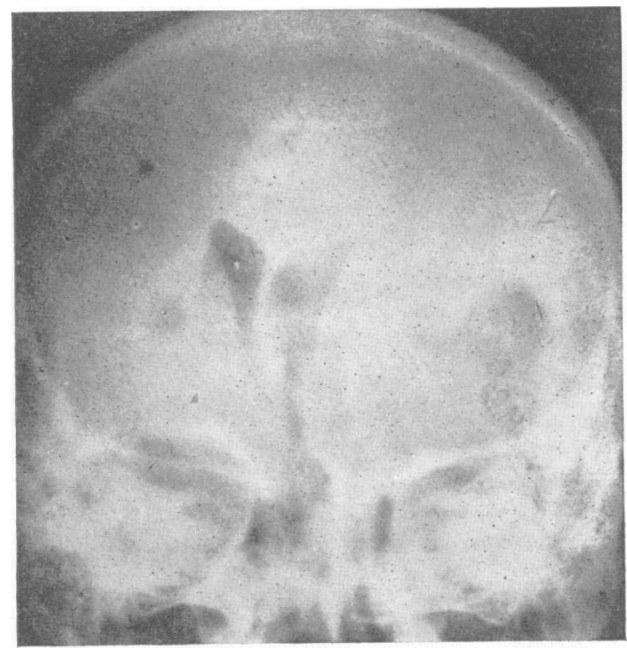

FIG. 3.-Ventriculogram, postero-anterior.

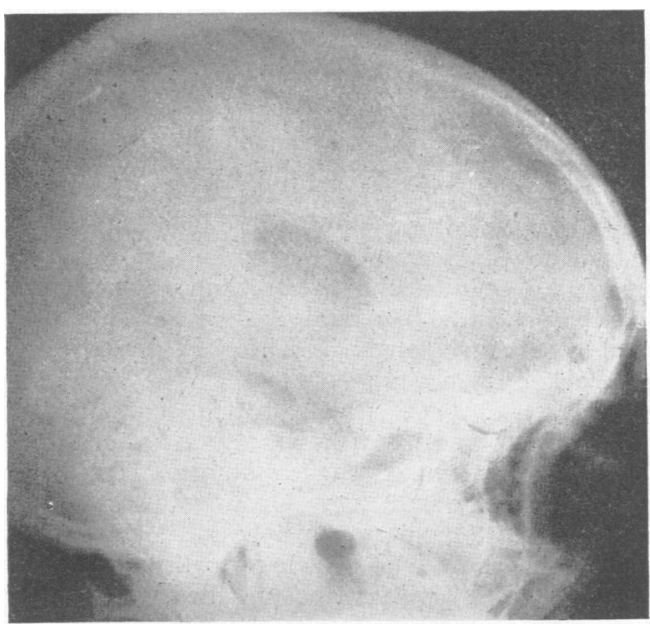

Fig. 4.-Ventriculogram, right lateral. 

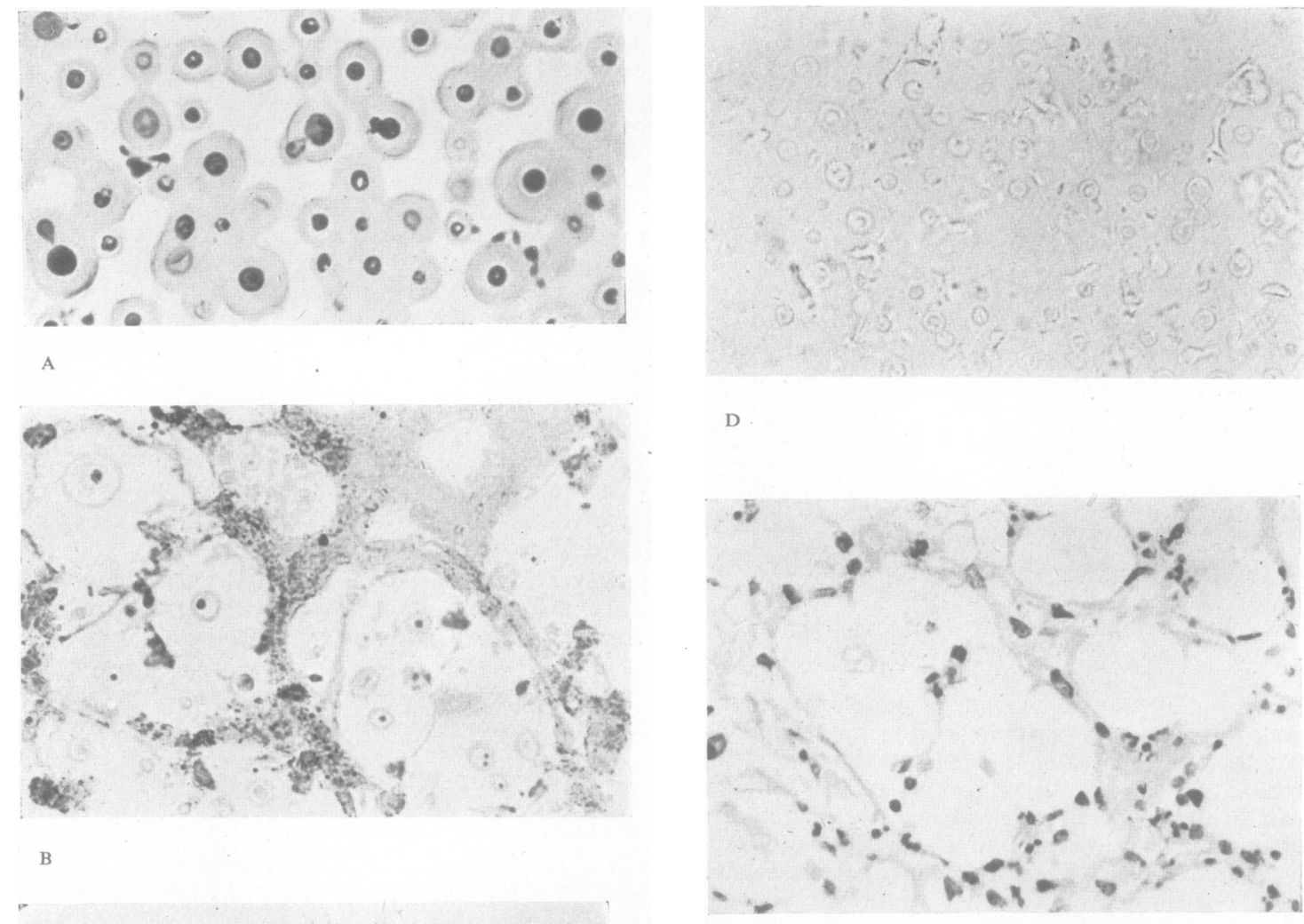

FIG. 5.-Neuropathology, Torula histolytica, $\times 450$

(A) aspiration from the lesion in the right thigh,

Gram stain ; .(B) same as (A) Sudan III stain ; (C) broth culture (subculture from agar) 36-hour Gram stain; (D) lymph node from the thoracic inlet of inoculated mouse, unstained smear ; (E) biopsy of the orbital lesion; haematoxylin eosin stain.

occasional budding and surrounded by a faintly bluestained homogeneous capsule. Smears stained with Sudan III showed the Gram-positive spheres lined by a highly refractile membrane, and one or more Sudan positive oil dots were enclosed. Occasionally the capsule showed a radial structure and was also lined by a highly refractive membrane. No mycelia and no ascospores were seen. The size of the cells, including capsule, varied from 7 to 24 microns, the Gram-positive spheres measuring 3 to 15 microns (Figs. 5A and B). Smears from the orbital wound showed a similar picture, but the spheroidal cells were scantier and there was marked admixture of polymorphonuclear leucocytes and bacteria.

Culture (Fig. 5C). - Sabouraud glucose and maltose agar ( $p \mathrm{H} \mathrm{6.0)}$ were inoculated with material aspirated from the lesion in the thigh and incubated at $37^{\circ} \mathrm{C}$. and at room temperature, $30^{\circ}$ C. Small, round, flat, translucent colonies with smooth surfaces appeared on the third day. becoming elevated and opague, and measuring up $6 \mathrm{~mm}$. in diameter on the sixth day. Growth was better at room temperature. Subculture on Sabouraud broth (glucose and maltose) showed no pellicle formation, but the broth was slightly turbid with a deposit at the bottom, and there was a ring at the junction of the fluid and container. Agar cultures and broth cultures (Fig. 5c) showed the same type of cells: small Gram-positive spheres 3 to 7 microns in diameter, with occasional budding, no mycelium formation, and no ascospores. A capsule was not visible in young cultures. Cultures kept dry and cold for three months showed no ascospores, but the outlines of a narrow capsule were visible.

Biochemical reactions. - There was no fermentation and also no acid formation in peptone sugars (glucose, mannite, lactose, sucrose, dulcitol, lævulose and maltose, initial $p H$ 7.0). Gelatine was not liquefied.

Penicillin sensitivity.-There was no inhibition of growth in buffered broth cultures despite the fact that the penicillin content was raised to 50 units per c.cm. Plate culture on tellurite medium examined with the Heatley cup method showed no growth in the cup, and there was also a $5 \mathrm{~mm}$. halo with complete suppression of growth, the penicillin concentration being 20 units per c.cm.

Diagnosis.-The organism was classified as a fungus of the genius Cryptococcus, morphologically resembling Freeman's type III of Torula histolytica, the biochemical reactions and the general behaviour in culture indicating its close similarity to Cryptococcus psychrofilicus (Nino,1930).

Animal inoculation.-Material from the lesion in the right thigh was injected subcutaneously and intraperitoneally into two mice immediately after aspiration, less 
than $1 \mathrm{c.cm}$. being injected altogether. In addition, a pure subculture of the organism in 36-hour glucose broth was used for animal inoculation, $2.5 \mathrm{c.cm}$. of the broth being injected subcutaneously and intraperitoneally into two guinea-pigs, and $0.5 \mathrm{c} . \mathrm{cm}$. subcutaneously and intraperitoneally into two mice. In the first group (direct inoculation) one mouse died after 24 days, showing signs of respiratory distress. The mesentery was thickened and the mesenteric lymph nodes were enlarged. There was also great swelling of the lymph nodes at the thoracic inlet, possibly producing pressure on the trachea. Smears from the lymph nodes showed the same organism as in the human lesions, but in general the cells were somewhat smaller and did not exceed 15 microns. A section of a lymph node from the thoracic inlet showed that lymphatic tissue had been largely replaced by fungus cells. No pathological findings were visible in the remaining viscera. The second animal developed a swelling the size of a hazel-nut at the site of the injection into the subcutaneous tissue of its belly, but there was no disturbance of general health and the animal was killed after two months, the subcutaneous swelling showing fungus cells in large numbers.

In the second group, inoculated with broth culture, both guinea-pigs and one mouse died after 36 hours, the material injected subcutaneously having been already resorbed. The peritoneal cavity contained turbid fluid, most of the fungus cells were degenerating, and there was an admixture of polymorphonuclear cells. Some of the fungus cells, however, were not only well preserved but already showed a well-developed capsule. The second mouse died after 22 days and showed the same picture as the directly inoculated animal, the fungus cells being of the same size and showing a well-developed capsule (Fig. 5D).

The lesions of the experimental animals were analogous to the human lesions, the fungus which had lost its capsule in a 36-hour broth culture as well as in agar culture having regained its capsule after 36 hours in the experimental animals. As a rule the organism was smaller in the experimental lesions than in the human tissue.

Histology.-Examination of material removed from the orbit showed vascular granulation tissue with focal and diffuse round-cell infiltration, the inflammatory cells being lymphocytes, histiocytes, and polymorphonuclear; but the bulk of the tissue consisted of faintly staining fungus cells suspended in a meshwork of reticulum fibres. The fungus cells were shrunken, due to fixation (Fig. 5E).

The meningeal tumour was firmly attached to the inner surface of the dura mater, and a narrow strip of cerebral cortex was found adherent to the convexity in places. The growth was of firm consistency, its cut surface being glassy grey and exhibiting a few mucoid cysts. Histological examination showed a picture similar to that of the orbital lesion, but isolation of single fungus cells was less complete, large clumps being separated only by bands of collagenous tissue. Cystic areas, showing only a fex fungus cells in the centre and marked foreign-body reaction with foreign-body giant cells in the periphery, were seen. Degenerating fungus cells were also seen within the foreign-body giant cells. The dura mater showed hyaline thickening, with giant capillaries, but there was no evidence of fungus cells in the dura mater itself or on its outer surface. At the point where the tumour came into relation with brain substance it was limited by a dense layer of fibrous tissue, the adjacent cerebral cortex showing partial softening with plump astrocytes and very marked perivascular round-cell infiltration. There was no evidence of perivascular extension into the brain substance. The histological findings were identical with those described in torula granuloma and torula meningo-encephalitis, the bulk of the lesions being formed by fungus cells, the tissue reaction contributing very little to the volume of the growth.

\section{Discussion}

The meningeal lesion of this case presented itself clinically and radiologically as an intracranial tumour, the classical syndrome of torula meningoencephalitis being absent. Tumour-like lesions of the meninges and of the brain are extremely rare complications of torula infection. Swanson and Smith (1944) reported two cases and found only three cases in the literature. In the second case of Swanson and Smith, in which the tumour was situated in the cerebellum, the cerebrospinal fluid was examined before operation; the pressure was $350 \mathrm{~mm}$. and there were six cells per c.mm., the protein being $184 \mathrm{mg}$. per $100 \mathrm{c.cm}$. and the sugar content $80 \mathrm{mg}$. per $100 \mathrm{c.cm}$. No torulæ were seen on direct examination, and cultures were repeatedly negative. In our case also no increase of cells was noted before operation, and no torulæ were seen in the spinal fluid, while two cultures remained sterile. This indicates that circumscribed torula granuloma of the meninges is not necessarily associated with diffuse meningitis. Signs of intracranial tumour in a case of proved systemic torula infection, and also in the absence of specific spinal fluid changes, are in all probability due to circumscribed torula granuloma. Swanson and Smith recorded the granuloma at operation as resembling . an ependymoma. The tumour in our own case looked like a meningioma. In Swanson and Smith's case, and also in our own, the tumour was sharply demarcated from the brain substance and there was no histological evidence of perivascular invasion. The anatomy of the lesion suggests that occasionally torula granuloma may be removed, with resultant cure.

According to Freeman (1930) only the large torulæ (type III) produce purely meningeal lesions Examination of our own case, and comparison with the two cases of Swanson and Smith, indicates that the circumscribed torula granuloma is formed by the large variety of the organism; hence the finding of large torulæ in systemic lesions is possibly of some prognostic significance. Animal inoculation carried out in our own case indicated that the actual size of the organism is to some extent determined by the nature of the host.

Torula meningo-encephalitis in man is usually associated with pulmonary lesions. But in monkeys successfully inoculated by the - cisternal route (Tronconi, 1934) the lungs may be macroscopically normal. The respiratory passages are usually considered to be the portal of entry of the infection, but evidence of this is scanty in man. Two cases reported by Semerak and quoted by Freeman showed the oldest lesions to be in the region of the Gasserian ganglion, and this was interpreted as an extension of the infection from the pharynx. Freeman, with Weidmann, found torulæ in the meninges of experimental animals three days after intranasal 
inoculation. In our case a pulmonary lesion, possibly due to torula infection, was also present, but this lesion at no time showed any signs of activity. Involvement of the frontal sinus was prominent at an early stage, and secondary extension from this focus into the orbit and meninges seemed probable on clinical and pathological grounds.

\section{Summary}

A case of torula infection presenting systemic lesions and meningeal involvement is reported. The meningeal focus produced the clinical and radiological picture of a space-occupying lesion situated in the left frontal region. At operation a globular tumour was found sharply demarcated from the brain substance and attached to the dura mater, the consistency and colour being that of a meningioma. The histological examination showed the typical picture of torula granuloma. Specific cerebrospinal fluid changes were absent before and after operation.

The causative organism was isolated from the systemic lesion of the right thigh before there were any signs of a cerebral tumour. On morphological grounds the organism was classified as type III of Torula histolytica Freeman.

We are indebted to the Director of Medical Services, India Command, for permission to publish the paper, and to Col. Faruki and Col. Sachs for their interest in the work. Thanks are also due to Prof. Kumara for permission to use the microphotographical equipment of the Agricultural College, Poona.

\section{REFERENCES}

Dodge, C. W. (1935). "Medical Mycology," C. V. Mosby Co., St. Louis.

Freeman, W. (1930). Trans. Amer. Neurol. Ass., 56, 203. Kuetzing (1833). Algarum aquae dulcis Germanicarum Decas, III, No. 28. (Quoted from Dodge's "Medical Mycology".)

Nino, F. L. (1930). Bol. Inst. clin. Quirurg. Univ. Buenos Aires, vol. 5. (Quoted from Dodge's "Medical Mycology".) (1938). Inst. Clin. Cir. Buenos Aires, Boletin No. 117, p. 591.

Stoddard, V. L., and Cutler, E. C. (1916). “Torula Infection in Man." Baltimore.

Swanson, H. S., and Smith, W. A. (1944). Arch. Neurol. Psychiat., Chicago, 51, 426.

Tronconi, V. (1934). Riv. Patol. nerv. ment., 44, 32. 